\title{
The Implementation of a Tele-Homecare System with Service Oriented Architecture and HL7 Message Transmission Standard
}

\author{
Ching-Sung Wang* \\ Department of Electronic Engineering, Oriental Institute of Technology, Taipei, Taiwan \\ *Corresponding author: ff020@mail.oit.edu.tw
}

\begin{abstract}
Received January 09, 2013; Revised February 06, 2013; Accepted February 15, 2013
Abstract Because of the continuously growing elderly population and annually declining birth rate, the population structure faces major changes, creating problems regarding medical care and healthcare. To address these problems, this study proposes a tele-homecare system that combines wireless sensors and transmission technologies to provide simple physiological data analysis and remote health management consultation services. This system collects measured data, including heart rate, blood oxygen level, blood pressure, and electrocardiograph results from various physiological measuring instruments using Bluetooth wireless technology to transmit and save these data via the Internet to a remote database. The tele-homecare platform is designed with Web architecture of Smarty modular and provides services to users of all levels. The health management interface of the personalized service-oriented architecture (SOA) provides real-time physiological monitoring and analysis of physiological parameters, allowing users to inquire about their health information using mobile devices or personal computers. This study anticipates that by using Health Level Seven (HL7) as the unified and standardized electronic medical record and Extensible Markup Language (XML) as the data conversion format to provide expandable and integrated medical information services on the Internet and exchange medical histories with other medical institutes, medical resources can be shared. The combination of this system and information from medical institutes allows the integration of the patients' medical records with their daily physiological measurements. The integrated data can be used as a diagnosis reference for physicians, reducing the time required to modify prescriptions and enabling the nursing staff to fully understand the patients' physiological conditions. This reduces burden on medical personnel and family members providing long-term care, and ultimately improve the quality of medical services and reduce costs.
\end{abstract}

Keywords: Healthcare Integrated Systems, Serviced Oriented Architecture (SOA), Enterprise Systems, Health Level 7, Extensible Markup Language (XML)

\section{Introduction}

In recent years, Internet and communication technology have been developed rapidly $[1,2,3,4,5]$. Techniques that combine engineering and medical advice in the development of homecare monitoring devices have become increasingly advanced in recent years. Numerous countries are attempting to apply information technologies totelecare, anticipating that by integrating medical care and technology, such as providing urgent notifications of abnormal conditions, the significant costs of medication reminders, recording and monitoring of physiological conditions, remote video communication, medical consulting, and medical expenses and staff can be reduced. Therefore, by combining the Internet and information technology to introduce the caring information system, the personalized home health management system, and a method of collecting users' daily assessment and measurement data via wireless transmission, the elderly can conduct physiological tests on themselves in the comfort of their own homes. The Web-based personal interface enables the elderly to receive good care at home, to stay informed about their current physiological status, to access their own health information, and to contact medical personnel and their families whenever an abnormal physiological condition occurs; this not only allows the elderly to better understand their own physiological conditions, but enables their relatives living away to be fully aware of the elderly person's conditions via the Internet. Professional healthcare staff could also receive a record of the seniors' daily test results. Receiving a copy of their records not only helps staff to better understand the conditions of the elderly when they receive treatments, it also helps staff to better understand the seniors' at-home daily physiological conditions. The system will provide a more complete picture of the patients' conditions on a 24 hour basis, increasing the accuracy of diagnosis and improving the quality of healthcare.

Therefore, the tele-homecare system should equip with various functions, such as wireless medical device, health surveillance, warning for abnormal condition, medical statistics integration. This study focuses on the demands of providing tele-homecare, designing remote physiological measurement devices, data recording equipment, and devices for real-time physiological 
monitoring, and provides users with wireless physiological measurements that can be uploaded and saved on the Internet. Remotely located users or medical staff can connect to the database anytime and anywhere, and access information on the users' present physiological conditions. Medical staff could also provide relevant medical counseling and advice to users according to the measured data. This system comprises the following:

1. A tele-homecare system designed to allow users to manage their own health.

2. Integrated and various monitoring devices that provide tele-physiological measuring via Bluetooth wireless transmission.

3. Web browsing health monitoring that allows users access to their personal physiological information.

4. Electronic medical records that enable theinformation to be shared information between the homecare system and medical institutes.

\section{System Integration}

The special features of each section in the system must be integrated first when designing this system to ensure the system implementation is complete and reliable. The special features of each section are as follows:

\subsection{Service-oriented Architecture (SOA)}

SOA is an architectural model comprised of standardized units of Web services technology. The aims of SOA are to establish a flexible and reusable integrated interface for enterprises, schools, and network service providing units to improve communication between the internal and external units, such as internal applications, users, and departments, and to enhance internet services [6]. SOA replaces the previous systematic development methods, which were object-oriented components, and implements the applications and resources using Web services. The calling and saving between system components is done via the interface, and standardized messages are used to exchange information. The combination of components can easily be changed according to the service processes of the entrepreneur at any time. SOA has been used to integrate heterogeneous platforms, for example, NET of Microsoft and J2EE of SUN can communicate and exchange saved information via standard protocols, such as HTTP, SOAP, and XML on Web services, reducing the cost of developing a system.

\subsection{Health Level Seven}

Health Level Seven (HL7), which was established in the U.S. in 1987, emphasizes the development of a medical information environment and electronic data exchange standards. HL7 transfers information of the hospitalization, discharge, and transfer of patients, sends queries regarding clinical observations, and establishes standard documents for delivering hospital billing data. HL7 simplifies the integrations between applications, reducing the costs of system development and establishing standards for medical information. Since the release of HL7 version 1.0 in 1987, v2.0, v2.1, v2.2, v2.3, v2.3.1, and v2.4 in 2000 have been successfully released. The version used currently is v3.0. Similar to HL7 (v2.x), HL7 (v3) provides a standard for information exchange between information systems in medical institutes. HL7 messages were first established in 1987. Because the number of members and users from different fields is continuously increasing, HL7 determined that a complete change to the medical systems was required. Thus, HL7 (v3) invested significant effort to improve the processes and results of HL7 (v2) to reduce the cost and time spent developing medical interfaces $[7,8]$.

Presently, numerous countries recognize HL7 as the standard system for exchanging hospital information. HL7 defines the grammar and semantics, specifies the labeling, and increases the ability to exchange medical information via XML (Extensible Markup Language.) Adopting the HL7 data format, using XML for exchanging information between medical resource integration modules, and unifying the information exchange structure can increase compatibly with other systems, enabling information to be shared with other medical institutions, care centers, and software and hardware development organizations.

\subsection{Extensible Markup Language}

Extensible Markup Language (XML) was developed by the World Wide Web Consortium (W3C) and is a marking language used for marking electronic documents that contain structural information. XML is a subset of SGML, which was formulated according to SGML format, and is a simplified version of SGML.XML eliminates the disadvantage of SGML being too complex. XML format is similar to HTML; however, the disadvantages of HTML being too simple to be expanded and unable to process large amounts of information were addressed by expanding the use of the Internet. Therefore, XML is not used to terminate the use of HTML, to replace SGML, or to abolish old standards; XML is used to set new standards and define a new marked language. XML is a metalanguage that describes marked language. In other words, XML provides the possibility of a defined mark and the probability of structural ratios between marks. No preset marks exist; therefore, no preset semantics are to be used to formulate and to generate other marked languages. An XML document comprises Readme information, which already contains related information such as data profile, file descriptor table, and additional defined data types. In contrast, HTML is not a standard document with a descriptive structure; it can present the information in the correct format but cannot preserve the applicability of the document as XML does. Therefore, the majority of manufacturers use XML standard, and develop agent programs of the database to conduct data exchange.

\subsection{Extensible Stylesheet Language}

Extensible stylesheet language (XSL) is an Extensible Markup Language designed to express formats. Due to its expandability, XML does not have marks that display related formats; XSL can choose and screen data within the expandable formats and transform the data into other document formats such as HTML or PDF.

XSL can be divided into three parts: XSL transformations (XSLT), XSL formatting objects (XSLFO), and XML path language (XPath), all of which are XSL programs. The main function of XSLT is to transform data from XML to another format; making it an important component of XML based e-commerce, electronic data interchange, metadata exchange, and 
applications that require using the same data on different XML to represent the transformations between. XML is purely an application to transfer data from one system or program to another.

\subsection{Electronization of medical record}

Consider Taiwan, for example, the National Health Insurance system accelerated the popularization of computerized medical history in medical institutes, and laid a solid foundation for entering the electronic medical record (EMR) stage. A prerequisite for using electronic medical records is that the EMRs must possess legal effect in the place of written records. As required by law, all important contracts and documents must be in written form to possess legal power. The paper-free EMR is in electronic data format and not the traditional written format, and would inevitably encounter obstacles regarding legal testimony. To an enter EMR stage and ensure electronic medical procedures and information is effective, the legal status of EMR must be established; the adaptation of the Taiwan Electronic Signature Act is the crucial link. The authorities amended the Medical Care Act and provided measures for the production and management of EMR in medical institutes according to the authorization of parent law after Taiwan passed the Electronic Signature Act in 2001, thus making EMR legally effective [9].

The Taiwan Council for Economic Planning and Management also passed the sub-project "Accelerated implementation of an EMR system in medical institutes," adding to the "Acceleration for intelligent medical care program" by the Department of Health, Executive Yuan in 2001. The program was scheduled to begin from 2010 to 2012, and $80 \%$ of all hospitals and $70 \%$ of all clinics are expected to provide ERM within three years [10].

\subsection{Telemedicine}

The American Telemedicine Association defined telemedicine as "exchanging medical information via electronic communication to improve the patients' health conditions, educate patients and caretakers, and ultimately, to improve the quality of care for patients [11]. A broad interpretation of telemedicine is medical behaviors, such as consultation, note taking, and examination, conducted at a distance. A more generalized definition for telemedicine is remote electronic care. The major goals of remote electronic care are to improve personal health conditions, promote quality of life, ensure economic efficiency, establish sustainable health and society, develop a healthcare system, and to provide prevention measurements. The Department of Health, Executive Yuan, of Taiwan defined telemedicine as "the use of electronic communication and information technologies to provide or support remote medical care, patients and professional health-related education, public health, and health management [12]."

\subsection{AJAX}

AJAX [13] is an acronym for "asynchronous JavaScript and XML," and is a Web development technology for establishing interactive Web applications. Jesse James Garret, the author of AJAX, indicated that AJAX is similar to DHTML (Dynamic HTML) or LAMP (Linux +
Apache + MySQL + PHP); AJAX does not use a single technology, but a series of organic technologies, as detailed below:

1. XHTML + CSS (Cascading Style Sheets) to represent information

2. JavaScript to operate DOM (Document Object Model) for dynamic displays and interactions

3. XML and XSLT for data exchange and related operations

4. XML Http Request objects to perform asynchronous data exchange with the Web server

5. JavaScript to link everything together

A number of AJAX-based "derivative/composite" technologies are emerging, such as AFLAX (Javascript + Flash). AJAX applications support Web browsers developed by various manufacturers as execution platforms. Current browsers include: Internet Explorer, Mozilla, Firefox, Opera, Konqueror, Google Chrome, and Safari of Mac OS.

Traditional Web applications allow the user end to fill in a form, upon sending the form, a request is sent to the Web server, then the server receives and processes the incoming form and sends back a new Web page. This approach wastes a lot of bandwidth because most of the two pages before and after the HTML codes are the same. Because requests must be sent to the server to communicate each application, the response time depends on that of the servers', resulting in a much slower response of the user interface compared to native applications.

AJAX is different. AJAX applications can deliver and retrieve required information from the server using Simple Object Access Protocol (SOAP) or other XML-based Page service interfaces; it also and uses JavaScript to process responses from the server at the user end. Due to a massive reduction in the information exchanged between the server and the browser (approximately $5 \%$ of the original amount), the server can provide a faster response and a quicker processing of applications. In addition, many processes can be performed at the user end by the equipment that sent the request. Therefore, the process time required for Web servers is also reduced.

\subsection{Wireless Transmission}

Currently, a wide range of wireless transmission technologies are commonly used, such as Bluetooth, Wi$\mathrm{Fi}$, ZigBee, and RFID [14,15]. Considering the power consumption, interference, and real-time data transportation, the wireless transmission interface of this system uses the Bluetooth technologies which are also frequently applied to computers, mobile phones, and wireless transmission technologies of other small appliances.

The operation principle of Bluetooth is to transmit digital data and sounds via a $2.45 \mathrm{GHz}$ band. Each of the Bluetooth connection devices can transmit peer-to-peer or peer-to-many according to their conditions. When transmitting peer-to-peer, two Bluetooth devices will create a communication connection not disturbed by other devices. A communication connection allows up to eight Bluetooth devices to conduct data transfers. The effective transmission range is $10 \mathrm{~m}$ and can even reach as far as $100 \mathrm{~m}$. Bluetooth technology not only has a great transmission capacity (up to $1 \mathrm{MB}$ per sec), encryption 
can also be set for protection, where the frequency is changed 1600 times per min. Therefore, Bluetooth is less susceptible to electromagnetic interference [16].

\section{System Design and Architecture}

This study designs interactive and multi-functional Web interfaces for a tele-healthcare system using the Smarty Web template framework. The system uses serviceoriented architecture (SOA) and the standard structure of HL7 to exchange health information, the ease of information exchange with other medical record systems is also considered. Additionally, this system transmits the users' physiological measures such as pulse, blood oxygen levels, blood pressure, and electrocardiograph results to the system via Bluetooth wireless transmission technology.

\subsection{Integration of Physiological Measurement Devices}

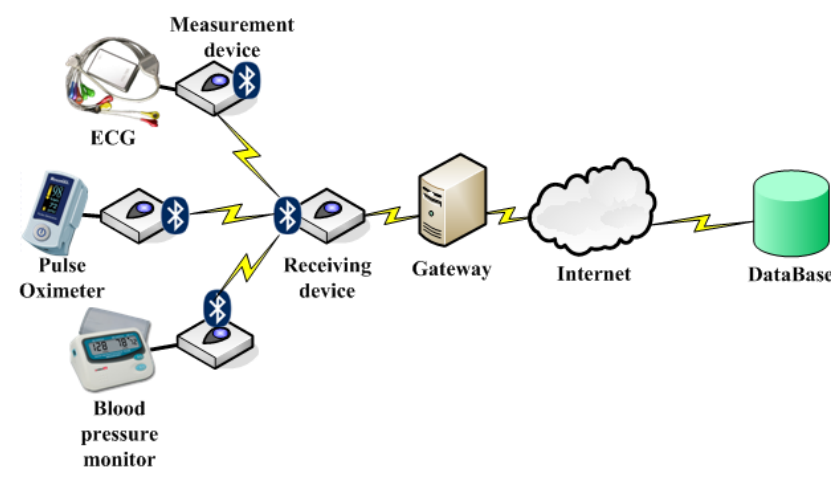

Figure 1. Measurement integrating device

The current physiological measurement devices on the market have stable performances. However, the designs and production of the measuring devices by various manufacturers all have their own transmission protocols and not all products can transmit the measured results to personal computers for management and further use. Therefore, a device connecting measurement devices was designed (Figure 1) to capture the measured data, such as pulse, blood oxygen levels, blood pressure, and electrocardiograph results. The data parameters of each device are then integrated and transmitted to receiving devices for further applications.

\subsection{Wireless Physiological Sensing Equipment}

The measured data captured by the integrating device can be transmitted wirelessly for the convenience of the users. However, to make homecare a ubiquitous service [17], enhance service advantage and convenience, and make tele-homecare more likely to be accepted depends largely on the security, stability, continuity, and expandability of the wireless communication mode.

The most widely used wireless transmission method on mobile devices and personal computers is Bluetooth. Bluetooth technology uses an open design, once devices certify each other they are able to communicate freely without limitations; they can also be used to create wireless connections between different devices. Therefore, Bluetooth wireless transmission technology was chosen for transmitting data to wireless physiological sensors.
This system captures the measurements of medical devices and transmits the information via Bluetooth; the information can be received via personal computers or smart devices and uploaded in XML format (as shown in Figure 2.)

\subsection{Tele-homecare system}

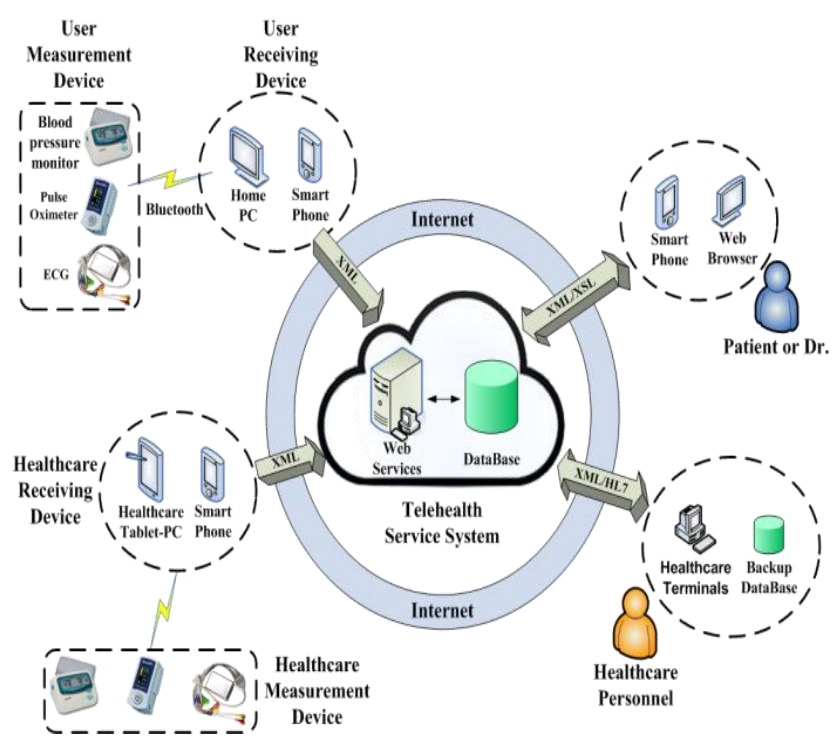

Figure 2. System schematic

The server of the tele-homecare system is responsible for receiving data uploaded by measurement devices, reprocessing the data, and saving the data to the corresponding database location. As for health management and telecare services, the server enables the users, the families, and the healthcare providing unit access to the users' physiological conditions at any time through the Internet using personal computers or smart devices $[18,19]$.

\subsubsection{Health Management Service Platform}

In addition to reducing the repeated design of a Web page, Web architecture using Smarty modular provides different functional interfaces according to different permissions. As shown in Figure 3, the health management service platform can be divided into three main structures: the data transfer module, the user module, and the healthcare unit module, and the different modules are assigned with different functions. An introduction to the functions provided by the health management service platform established in this system.

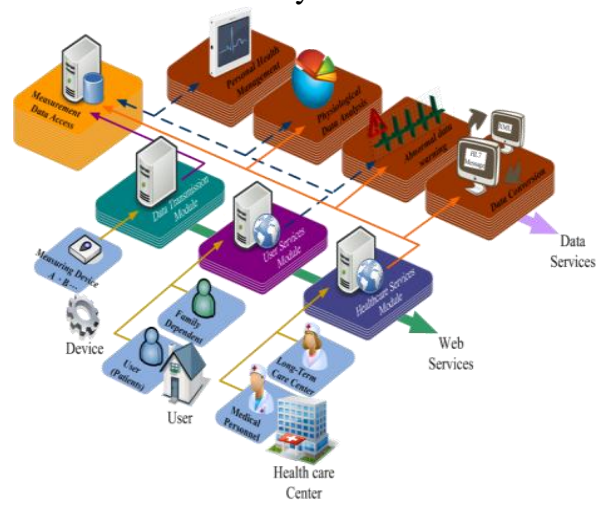

Figure 3. The platform architecture of the health management service 
1. Personal health management function provides users with autonomous health managing services. When at home, users can use frontend devices for physiological measurements, the measured data will then upload to the database of the telecare system via the Internet. The Webbased user interface allows users and their families to query personal physiological measurement data and related information from a Web page without having to install additional tools; the module also permits inquiries into the advice provided by medical staff.

2. The physiological analysis function is responsible for saving the physiological measurement data uploaded by users or patients to the database. This analysis function categorizes the users' records by month, saves the data, displays the data are in graphs, and monitors the uploaded physiological data. If the uploaded data are out of the range recommended by doctors or beyond the set values of the system, the abnormal data warning function will immediately warn the user's consulting physician via the interface, and will also display the abnormal data and related information of the individual to enable the physician to better understand the patient's physiological conditions $[20,21]$.

3. This system was designed to use an XML format to exchange medical information to share medical resources between medical units using other systems. The advantages of using XML for exchanging information are:

a. When processing repeated and shared information, a structured information format is advantageous for the exchange and processing of information.

b. XML does not set a restriction on the representation format.

c. The label element for the content of XML documents is independent of the communication protocol in principle. Therefore, the XML document is particularly suitable for use on the Internet and for global information network transmissions.

d. XML has many options to choose from in the editor application, middleware, and the application tools, which greatly enhances the efficiency and saves the costs of developing and managing an information system.

\subsubsection{Web Services System Implementation Process}

As shown in Figure 4, the system distributes the user to the assigned level once they log in. As the patient (user) logs on, the system distributes the patients to the user level and links the database for reading and analyzing the measured data. If abnormalities are observed in the measured data, for example, systolic blood pressure is higher than 120, diastolic blood pressure is higher than 80 , and the heart rate is greater than 100 beats/min or lower than 60 beats/min [22], the system reports the information of the patient showing abnormal readings to the medical staff module, the abnormal figures are also shown on the Web page as the patient (users) logs in.

When medical staff use the system, the system logs into the medical unit level, loads the module for medical staff, and searches for patients with abnormal data readings. Once abnormal data is found, the system notifies medical staff to contact the patient to check their condition and repeat and confirm the measurements. Abnormal measurements are also sent to the patient's family's system to notify them of the current condition of the patient. The ID of the patients showing abnormal measurements that day are displayed on the medical staff login page.

When the patient's family logs into the system, the system logs into the level for patients' families and uploads the patients' family module together with the patient's information. If the patient has abnormal measurements, the system notifies the families by displaying the abnormal figures on the family's login page.

\subsubsection{Share of Medical Resources}

EMR uses an XML data transmission format and can efficiently exchange information between users and medical institutes. The data transmission format varies according to the service receivers. The two major applications of XML documents in this system are shown in Figure 5.

1. Transform XML into HTML or PHP:

When the receiver of the XML document is a different device type or cannot directly read the XML file format, the system transforms the XML document via XSL technology. XSL transforms all data or parts of the data in the original file using XSLT and chooses the content using XPath; the final output data is either in HTML or PHP Web page format according to the users' needs.

\section{Transform XML into XML:}

Transforming one XML file format into another XML format is primarily used for exchanging information between two organizations or systems. For example, if hospital A and B are to exchange medical records but the two hospitals have different XML file formats, they can use XSLT to transform the XML documents into the standard HL7 format to facilitate data transfer.

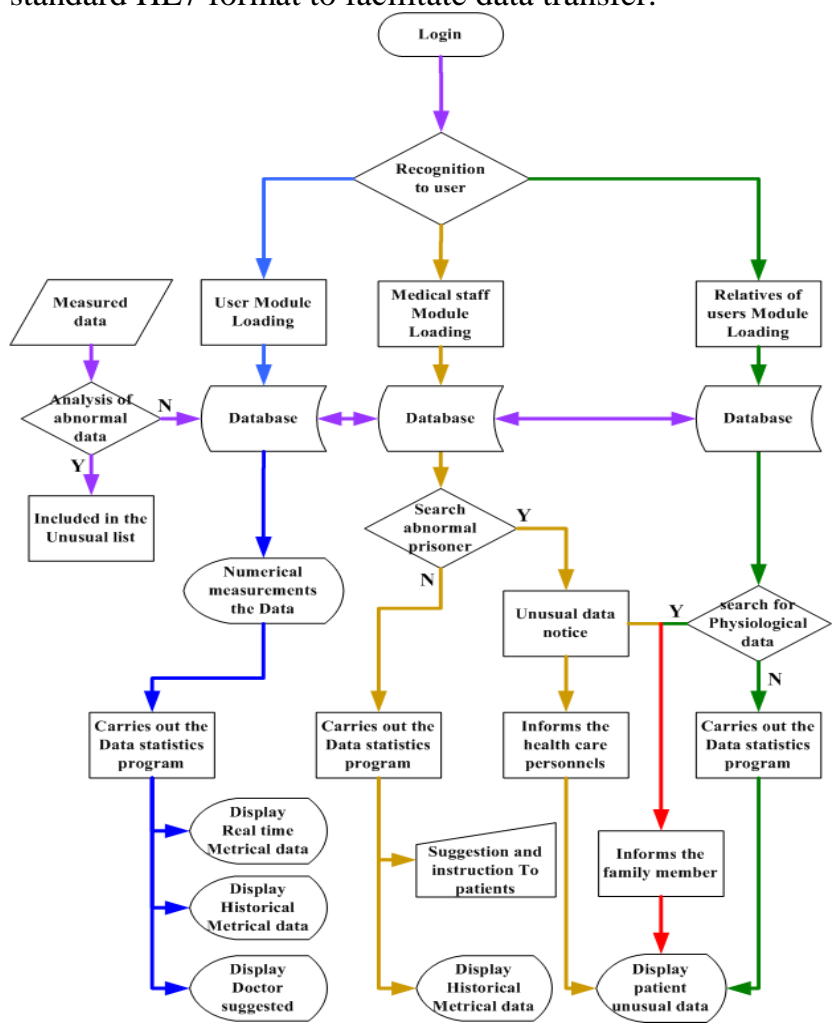

Figure 4. System flowchart

As shown in Figure. 6, information in XML format can be converted using XSL, enabling the source data to be displayed in various formats, such as a Web browser, data transmission between devices, and data conversion by 
medical institutes, according to different user requirements or user interfaces. Transmitting data in XML format and uploading information to the Web server enable the construction of thetele-healthcare service Web page in a modular manner. Planning and using different blocks and browsing interfaces according to the different user levels reduces the resources and time consumed redesigning Web pages.

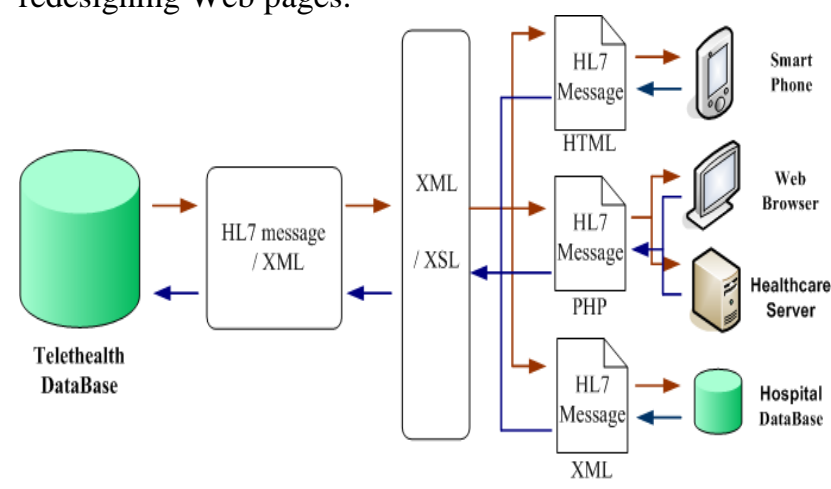

Figure 5. The transmission and transformation of information

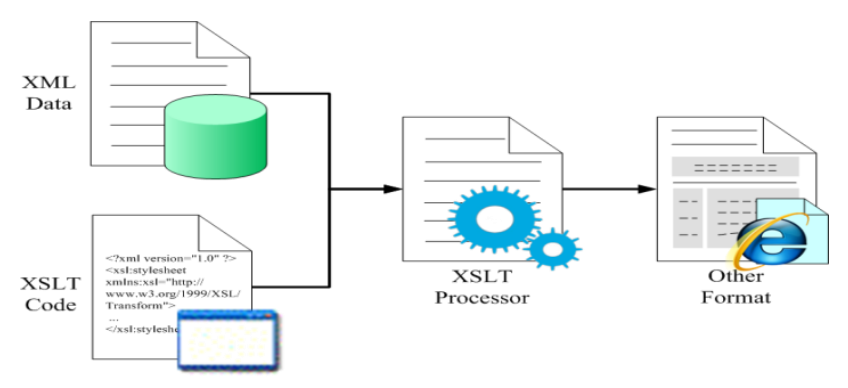

Figure 6. Schematic drawing of the conversion process

\section{Measurement Results}

This study finished establishing the tele-homecare system and is presently conducting actual physiological measurements and data analysis. The system implementation results and portions of Web page images on the Web end are detailed below.

\subsection{Design of a Wireless Physiological Sensor Device}

The complete wireless physiological sensor designed by this study. During the measurement process, users wear the designed wireless physiological measurement device and conduct measurements according to normal use. Upon measuring, the device extracts and uploads the measured data to the host database. Users can also link to the Internet during the measuring process and launch the health management service platform on the Web page. By choosing real-time physiological monitoring, all the measured data is shown immediately on the Web page (Figure 7).

\subsection{Health Management Service Platform}

Figure 8 shows the user interface of the modular health management platform (medical staff page). According to the user's login account, the user is assigned to different levels of user authority and guided to log into a specified module.

Figure 9 shows the login Web page of general users (patients). When users log in, the Web page first displays the various data measured that day. When the query measured data function is selected, users can choose other query functions for general users, for example, today's measurement data query, real-time measured data query, blood oxygen level and pulse record queries, and blood pressure record query.

Figure 7 shows the real-time measurement data query functions. When users (patients) use the measuring devices to measure physiological signals, the user end communicates with the server through the AJAX functionality of the Web page. The AJAX Web page and server communicate in the background at the user end, transmit data asynchronously, and display the user's measured data in the database.

Therefore, the Web page continuously reads the latest information and displays the information on the Web page in real-time. Users can also query previous physiological measurements, such as history of blood oxygen levels and pulse records, as shown in Figure 10. The system can display the measurement record of the date indicated by the user and show the blood oxygen levels and pulse (heartbeat) values using a histogram. This system provides one week, two weeks, and one month record query options for blood pressure record queries. The values of the selected range are displayed using a line chart, as shown in Figure 11.

\subsection{Transformation Processing of EMR to XML Format}

EMR is transformed into XML format by obtaining the required information from the Telehealth Service Database, and transforms the information into a uniform standard XML data through XML/XSL [23,24,25]. The output of the standard information formats can be divided into two types: EMR of the HL7 format, and XML format defined by hospital information systems (HIS). Meanwhile, the self-defined XML format is used for data transmission and information exchange, as shown in Figure 12.

\section{Conclusion}

This study established a tele-healthcare system that enables at home self-health management. This study also designed a Bluetooth wireless physiological measurement transmission device to measure the users' physiological condition. The Web services approach enables the saving and browsing of measurement records, which not only reduces problems related to having to install programs at the user end, but users can also monitor their measurement record through the Internet at any time. The crossplatform and cross-language features of Web services provide more flexibility in the use of the system and improve the promptness and convenience of message transmission between systems. This system uses XML for information transformation. Information is transformed into a format that conforms to the HL7 standard via XML, and therefore can exchange messages with various independent hospital information systems. This system 
uses Web services to provide real-time online healthcare, services allowing users and families to inquire simultaneously from different locations. Meanwhile, the system coordinates the requirements of medical staff and strengthens the interface and function of the Web page for medical staff to enhance the system's completeness. The tele-homecare system with aservice-oriented architecture and the HL7 information standard are expected to provide additional elderly people with better and more convenient healthcare services.

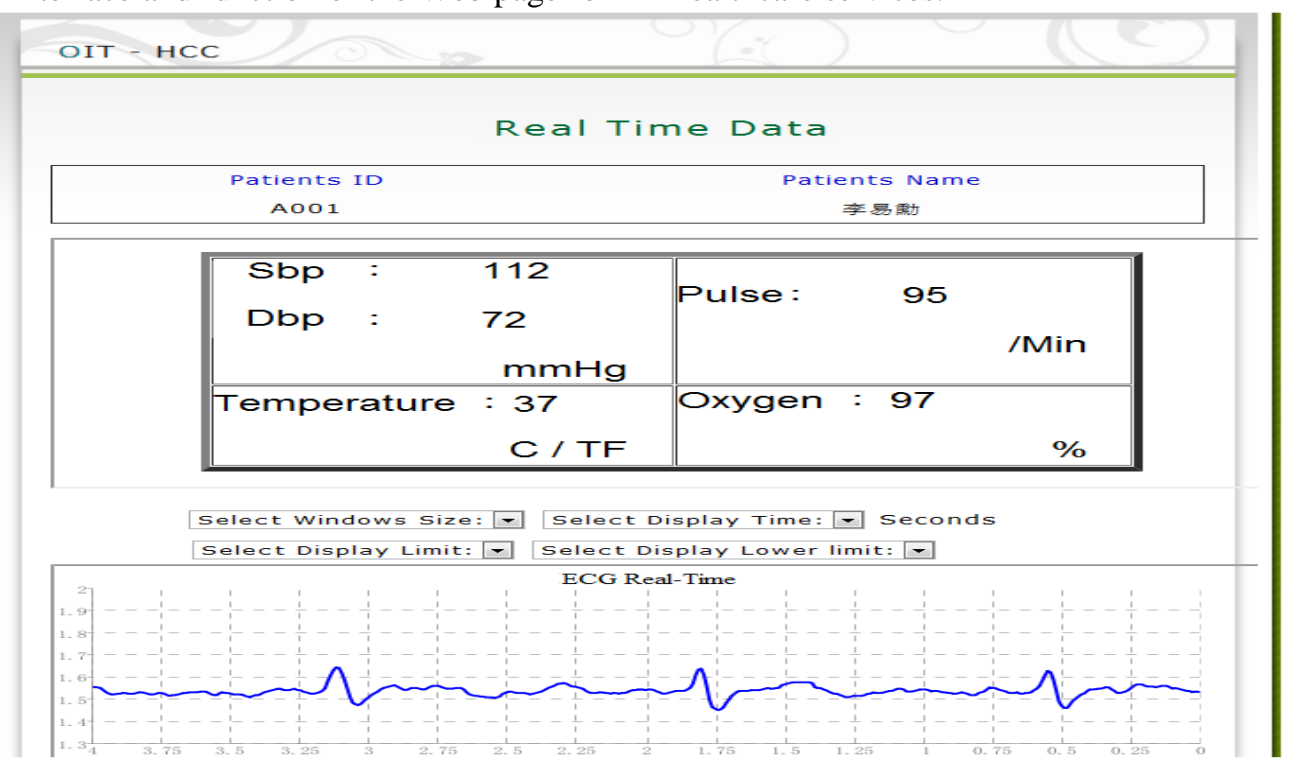

Figure 7.Real-time measurement data query functions

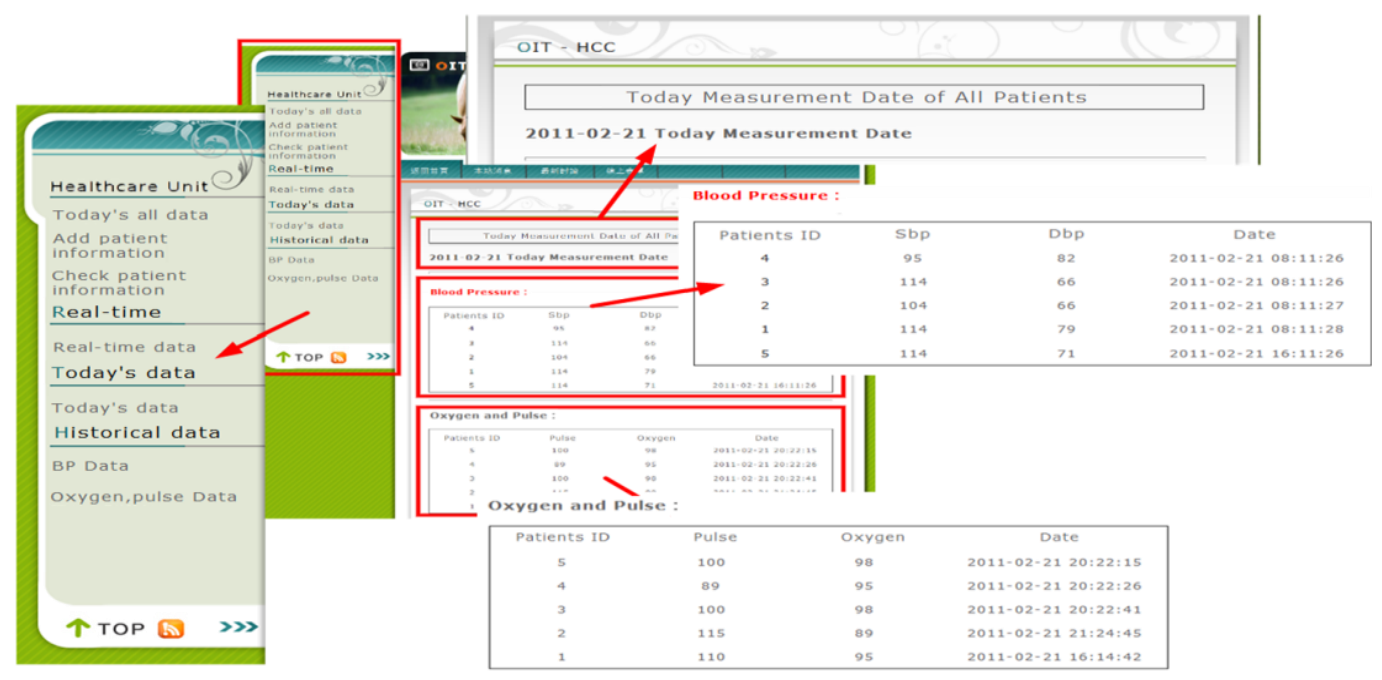

Figure 8. The user interface of the modular health management service platform (medical staff page)

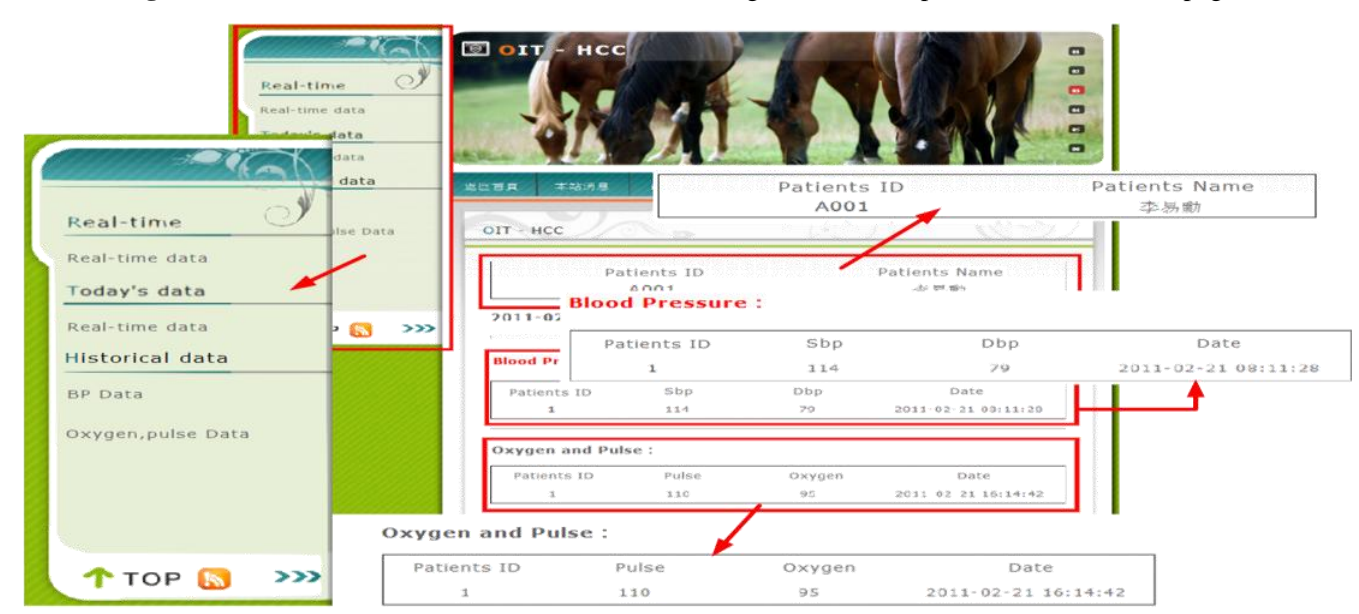

Figure 9. General user interface (patient Web page) 


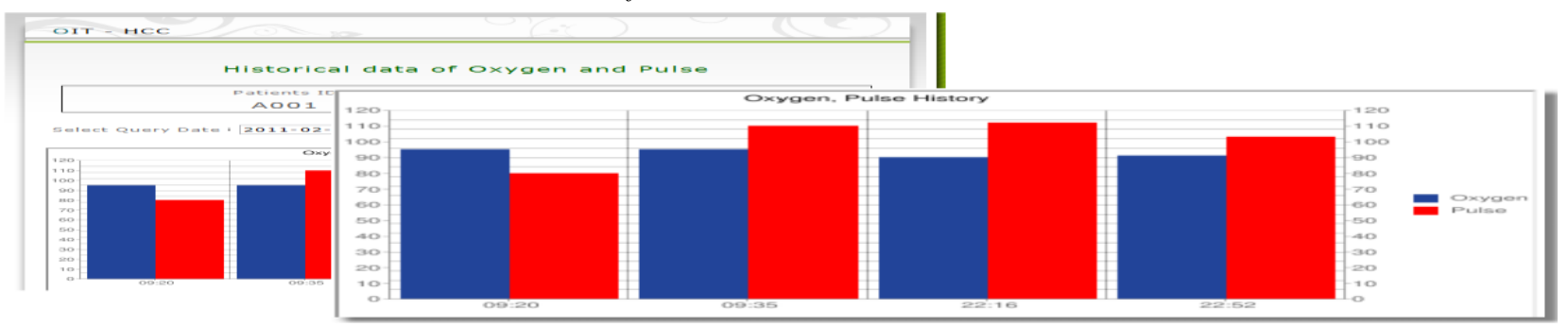

Figure 10. Blood oxygen and pulse recording query function

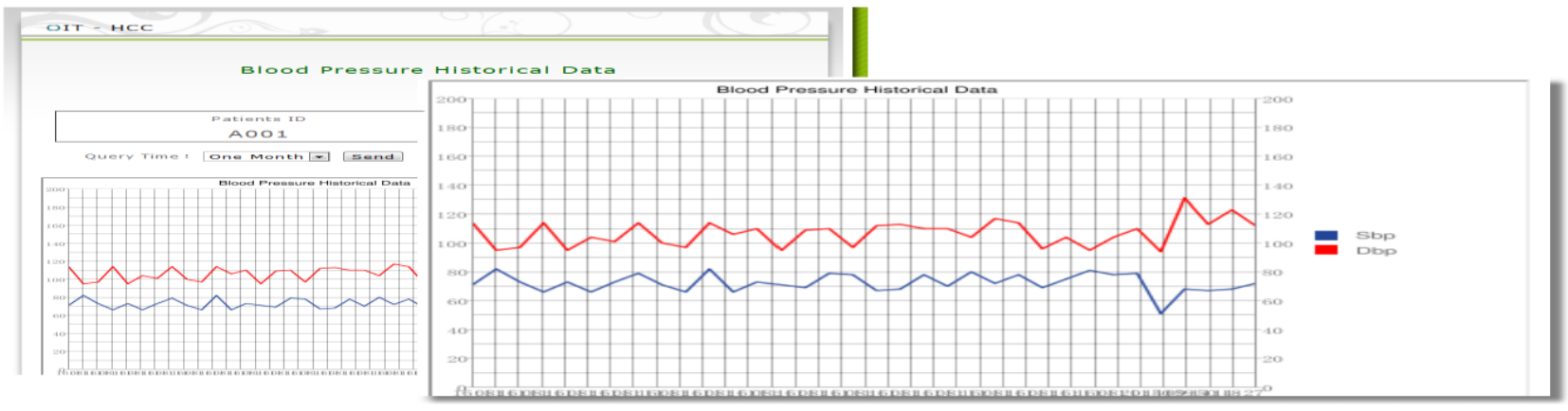

Figure 11. Blood pressure record query functions

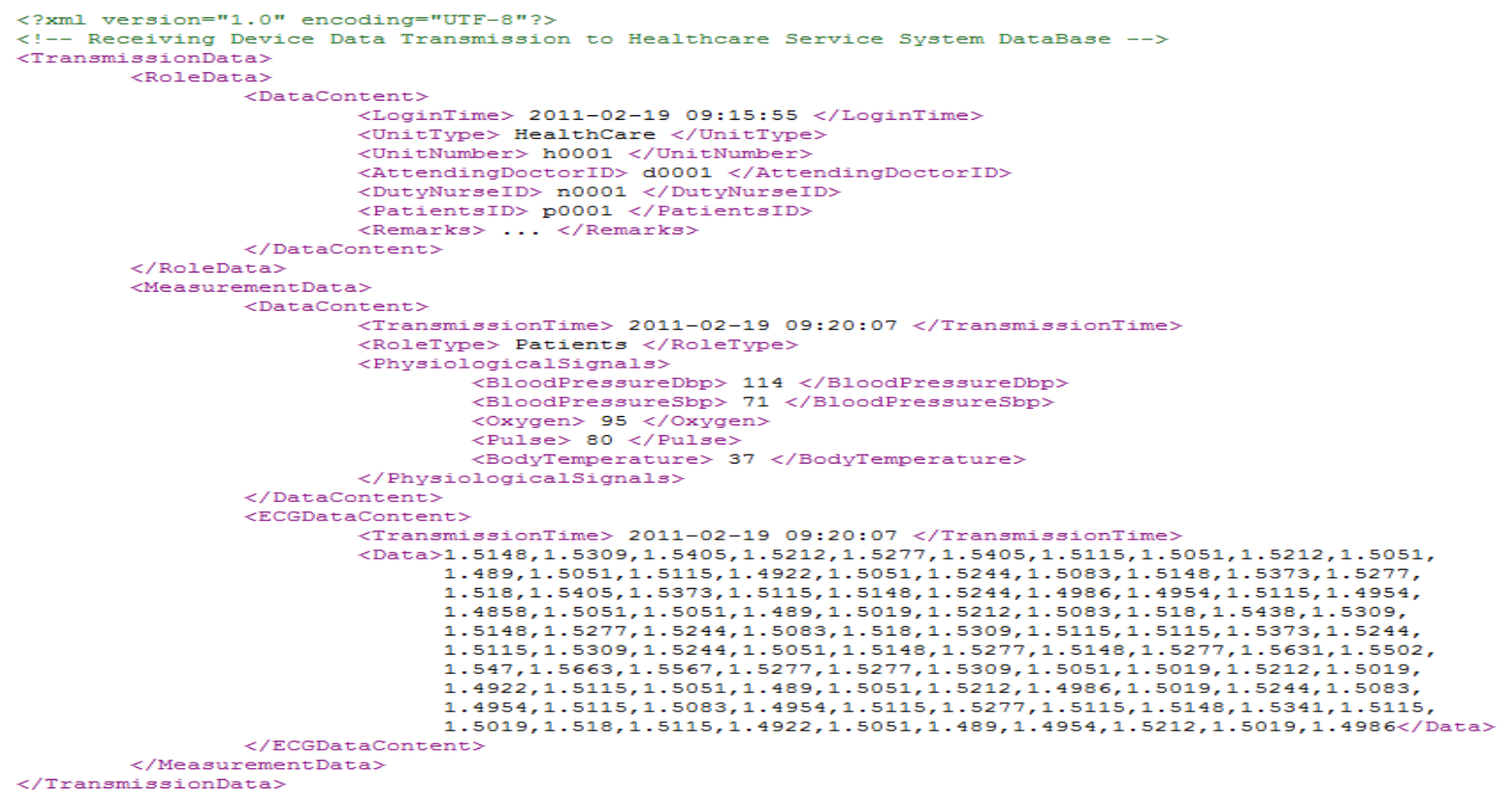

Figure 12. XML file format used for exchanging information

\section{References}

[1] Mietzner, R., et al. 2011. Horizontal and vertical combination of multi-tenancy patterns in service-oriented applications. Enterprise Information Systems, 5(1), 59-77.

[2] Xu, L. 2011. Enterprise systems: state-of-the art and future trends, IEEE Transactions on Industrial Informatics, 7(4), 630-640.

[3] Xu, E., et al. 2011. Development of an integrated medical supply chain information system. Enterprise Information Systems, 5(3), 385-399.

[4] Duan, L., et al. 2011. Healthcare information systems: data mining methods in the creation of a clinical recommender system. Enterprise Information Systems, 5(2), 169-181.

[5] Cao, X., and Yang, F. 2011. Measuring the performance of Internet companies using a two-stage data envelopment analysis model. Enterprise Information Systems, 5(2), 207-217.
[6] Bao-Jang Tseng. 2007 National Taiwan University, Computer \& Information Network Center, Division of Network Management, Electronic Newspaper, Vol. 0001.

[7] Health Level Seven. 2006 "HL7 Version 3.0 Edition 2006, http://www.hl7.org/about/," May.

[8] Kabak, Y.,et al. 2008 The Use of HL7 CDA in the National Health Information System (NHIS) of Turkey.

[9] Wen-Chin Chen. 2009 "Introduction to electronic medical record", Computing Center, Academia Sinica E-News, Vol. 23.

[10] Department of Health, Executive Yuan, (Taiwan), "Execution For electronic medical record".

[11] O. S. Adewale,2004 "An internet-based telemedicine system in Nigeria," International Journal of Information Management, Vol. 24,221-234

[12] World Medical Association Team. 2008 " World Medical Association Statement on Guiding Principles for the Use of Telehealth for the Provision of Health Car " Taiwan Medical Journal, Vol. 51. 
[13] Ji Jin Lin, Jin De Wang.2008 "Medical Management Information System Design Using UML and AJAX", St. John's University.

[14] Xu, L. 2011. Information architecture for supply chain quality management. International Journal of Production Research, 49(1), 183-198.

[15] Kumar, S., et al. Challenges with the introduction of radiofrequency identification systems into a manufacturer's supply chain-a pilot study. Enterprise Information Systems, 5(2), 235-253.

[16] Han-Hsyuan Wu. 2008 "The Development of a Remote HealthCare Unit Based on Embedded System - Monitoring of Location and $\mathrm{SpO} 2 "$, Graduate Institute of Electrical Engineering, National Taiwan University, Master dissertation.

[17] Kakousis, K., et al. 2010. A survey of software adaptation in mobile and ubiquitous computing. Enterprise Information Systems, 4(4), 355-389.

[18] Hamilton. 2005 L8S 4K1, Canada., Interoperability of Data and Knowledge in Distributed Health care Systems, IEEE computer society.

[19] V Chan, P Ray, N Parameswaran. 2007 "AMulti-Agent CollaborativeFramework for Mobile E-Health", Proceedings of the 40th Annual Hawaii International Conference on System Sciences.

[20] Peter L. et al. 2007 "Smart Homecare System for Health Telemonitoring", Proceedings of the First International Conference on the Digital Society.

[21] Kang, J. M., et al..2006 A Wrist-Worn Integrated Health Monitoring Instrument with a Tele-Reporting Device for Telemedicine and Telecare. IEEE Transactions on Instrumentation and Measurement, 55(5), 1655-1661.

[22] Taiwan Bureau of Health Promotion - Health 2010 Education Resource.

[23] Chi-Hsun Wu, et al.2006 "The Study on Web Services Based Health Level Seven for Electronic Health Record Exchange System" The Journal of Taiwan Association for Medical Informatics, Vol. 15,No. 2, June, 41-54.

[24] Chih-Hsun Chang, et al.2006"Research of Information Development for HL7 v3 - Example on Patients Data Requisition",The $5^{\text {th }} \quad$ Asia-Pacific and Cross-Strait HL7 Conference on Healthcare Information Standards.

[25] Robert H. Dolin et al., HL7 Clinical Document Architecture, Release 2.0,HL7 V3 Ballot Document. 